\title{
Different fundus imaging modalities and technical factors in Al screening for diabetic retinopathy: a review
}

\author{
Gilbert Lim ${ }^{1,2+}$, Valentina Bellemo ${ }^{2,3 \dagger}$, Yuchen Xie ${ }^{2}$, Xin Q. Lee ${ }^{2}$, Michelle Y. T. Yip ${ }^{3}$ and Daniel S. W. Ting 2,3,4,5*
}

\begin{abstract}
Background: Effective screening is a desirable method for the early detection and successful treatment for diabetic retinopathy, and fundus photography is currently the dominant medium for retinal imaging due to its convenience and accessibility. Manual screening using fundus photographs has however involved considerable costs for patients, clinicians and national health systems, which has limited its application particularly in less-developed countries. The advent of artificial intelligence, and in particular deep learning techniques, has however raised the possibility of widespread automated screening.

Main text: In this review, we first briefly survey major published advances in retinal analysis using artificial intelligence. We take care to separately describe standard multiple-field fundus photography, and the newer modalities of ultrawide field photography and smartphone-based photography. Finally, we consider several machine learning concepts that have been particularly relevant to the domain and illustrate their usage with extant works.

Conclusions: In the ophthalmology field, it was demonstrated that deep learning tools for diabetic retinopathy show clinically acceptable diagnostic performance when using colour retinal fundus images. Artificial intelligence models are among the most promising solutions to tackle the burden of diabetic retinopathy management in a comprehensive manner. However, future research is crucial to assess the potential clinical deployment, evaluate the cost-effectiveness of different DL systems in clinical practice and improve clinical acceptance.
\end{abstract}

Keywords: Artificial intelligence, Deep learning, Diabetic retinopathy, Fundus photographs, Retinal imaging modalities, Survey

\section{Background}

A growing global health problem related to diabetes mellitus, one of the world's fastest growing chronic diseases, is diabetic retinopathy (DR). This condition has been projected to affect 700 million people across the world within the next two decades [1]. Since one-third

\footnotetext{
* Correspondence: daniel.ting.s.w@singhealth.com.sg

${ }^{\dagger}$ Gilbert Lim and Valentina Bellemo contributed equally to this work.

${ }^{2}$ Singapore Eye Research Institute, Singapore National Eye Centre, Singapore, Singapore

${ }^{3}$ Duke-NUS Medical School, National University of Singapore, 11 Third Hospital Road Avenue, Singapore 168751, Singapore

Full list of author information is available at the end of the article
}

of diabetic patients have underlying DR, this would translate to approximately 250 million people suffering from DR by the year 2035 [2-4]. To meet this rapidly evolving and growing crisis, tools that are able to deal with this heavy workload quickly and efficiently are paramount in overcoming and tackling this leading cause of blindness across the world $[5,6]$.

Early detection of DR via population screening - associated with timely treatment - has been shown to have the potential to prevent visual loss in patients with diabetic retinal complications [7]. Many computer-aided algorithms for automated retina image analysis have been explored [8-12]. Since before the deep learning

C The Author(s). 2020 Open Access This article is licensed under a Creative Commons Attribution 4.0 International License, which permits use, sharing, adaptation, distribution and reproduction in any medium or format, as long as you give appropriate credit to the original author(s) and the source, provide a link to the Creative Commons licence, and indicate if changes were made. The images or other third party material in this article are included in the article's Creative Commons licence, unless indicated otherwise in a credit line to the material. If material is not included in the article's Creative Commons licence and your intended use is not permitted by statutory regulation or exceeds the permitted use, you will need to obtain permission directly from the copyright holder. To view a copy of this licence, visit http://creativecommons.org/licenses/by/4.0/ The Creative Commons Public Domain Dedication waiver (http://creativecommons.org/publicdomain/zero/1.0/) applies to the data made available in this article, unless otherwise stated in a credit line to the data. 
(DL) era, the development and application of such techniques has produced cost-effective tools for DR screening, $[13,14]$ and were crucial in the care of patients with DR and other diseases detectable from the retina such as glaucoma, age-related macular degeneration and retinopathy of prematurity [6, 15-17]. Several international research groups have worked on automatic retinal image analysis methods to detect, localize, or measure retinal features and properties, [18-20] such as automated segmentation and diameters measurement of retinal vessels [21].

In this review paper, we present some state-of-the-art DL systems for DR classification using fundus retinal images. We further aim to explain the machine learning (ML) techniques and concepts involved alongside a broad overview of major published works.

\section{Artificial intelligence in retinal analysis}

Artificial Intelligence (AI) is an attractive solution for tackling DR burden. ML is the subfield of AI that focuses on techniques and algorithms that learn to perform tasks without providing specific instructions, and the subset of ML that is DL has garnered particularly huge interest in the last decade $[5,22]$. DL was initially inspired by the neuronal connectivity of the brain, allowing it to process large amounts of data and extract meaningful patterns based on past experiences with the same input. Moreover, DL improved on prior and shallower artificial neural networks by being able to model data at various scale abstractions [23]. Specifically, deep convolutional neural networks $(\mathrm{CNN})$ has been at the forefront of this new wave of DL in medical analysis due to its remarkable ability to analyse images and speech with high accuracy. This has resulted in widespread applications in multiple medical specialties, including but not limited to ophthalmology, radiology and pathology [24-28]. CNNs have found particular success in these specialties due to their reliance on imaging data such as fundus photographs, radiological films and pathological slides [24-27].

The validation of such methods is key for demonstrating the robustness and applicability of DL technologies among clinicians, eye care providers, and biomedical scientists $[15,29]$. Large and rich sets of testing data are required for the development, as well as comprehensive expert annotations as reference gold standards [30]. To be effective, a high level of confidence in the agreement between the computer system and expert human readers is required. Sensitivity, specificity, accuracy, positive and negative predictive value, and AUC are common statistical analysis to assess the algorithm's output validity. Also, DL-based systems might serve as a promising solution to reduce human grading workload, and also serve as a cost-effective screening alternative for both highand low-resource countries [31-33].
Ophthalmology has been at the forefront of this revolution, and DL-based methods are expected to increasingly influence routine clinical patient care in the future $[16,33]$. In particular, Abràmoff et al. was the first group to obtain United States (US) Food and Drug Administration (FDA) approval for the use of a DL system in the diagnosis of DR from retinal images [34]. As for Google AI Healthcare, Gulshan et al. demonstrated high diagnostic ability for detecting DR whilst optimizing and minimizing the size of the training dataset required to achieve these results [35]. Ting et al. was able to translate this clinically by demonstrating the high performance of a DL-based system across multi-ethnic populations, despite not originally being trained with eyes of differential phenotypical characteristics, while being subject to non-optimal real-world image capture settings [26]. DL has also found success in detecting other ocular diseases from colour fundus photographs such as age-related macular degeneration, [36] glaucoma [37] and retinopathy of prematurity [38].

Despite many publications attesting to the robustness, reliability and accuracy of these DL systems in the detection of pathological states, and the support garnered from federal agencies such as the US FDA, translation into clinical practice has not been without its challenges $[16,39]$. Resistance to implementation has been largely due to the inscrutability of these algorithms [33]. This is due to the black box' concept that is evident in DL methods describing the ambiguity as to how these networks arrive at their conclusion [5]. Although this is a phrase commonly put forth during the analysis of the applications of DL systems, it holds significant weight in the field of medicine, where accountability for incorrect decisions weigh heavily, and where the patients' and physicians' trust is necessary for acceptance of a novel method [16]. That said, there exist methods that are introduced that help to address this issue, including saliency heatmaps that provide a visual representation of regions that DL systems consider in making a decision, or feature attributions where values are assigned to features and those with higher values suggest areas that are critical to the prediction by the model [40-43]. Such methods provide a certain reassurance with DL implementations, and allow for further translational progress.

\section{Main text}

\section{Retina fundus imaging modalities}

Fundus imaging is an established modality for retinal imaging, and the detection of DR from fundus images has a long and rich history in retinal analysis [44]. Fundus imaging is defined as the process whereby reflected light is used to form a two dimensional representation of the three dimensional retina, the semi-transparent, layered tissue lining the interior of the eye projected onto an imaging plane [45]. Figure 1 shows different levels of DR severity from retinal colour fundus images and Fig. 2 


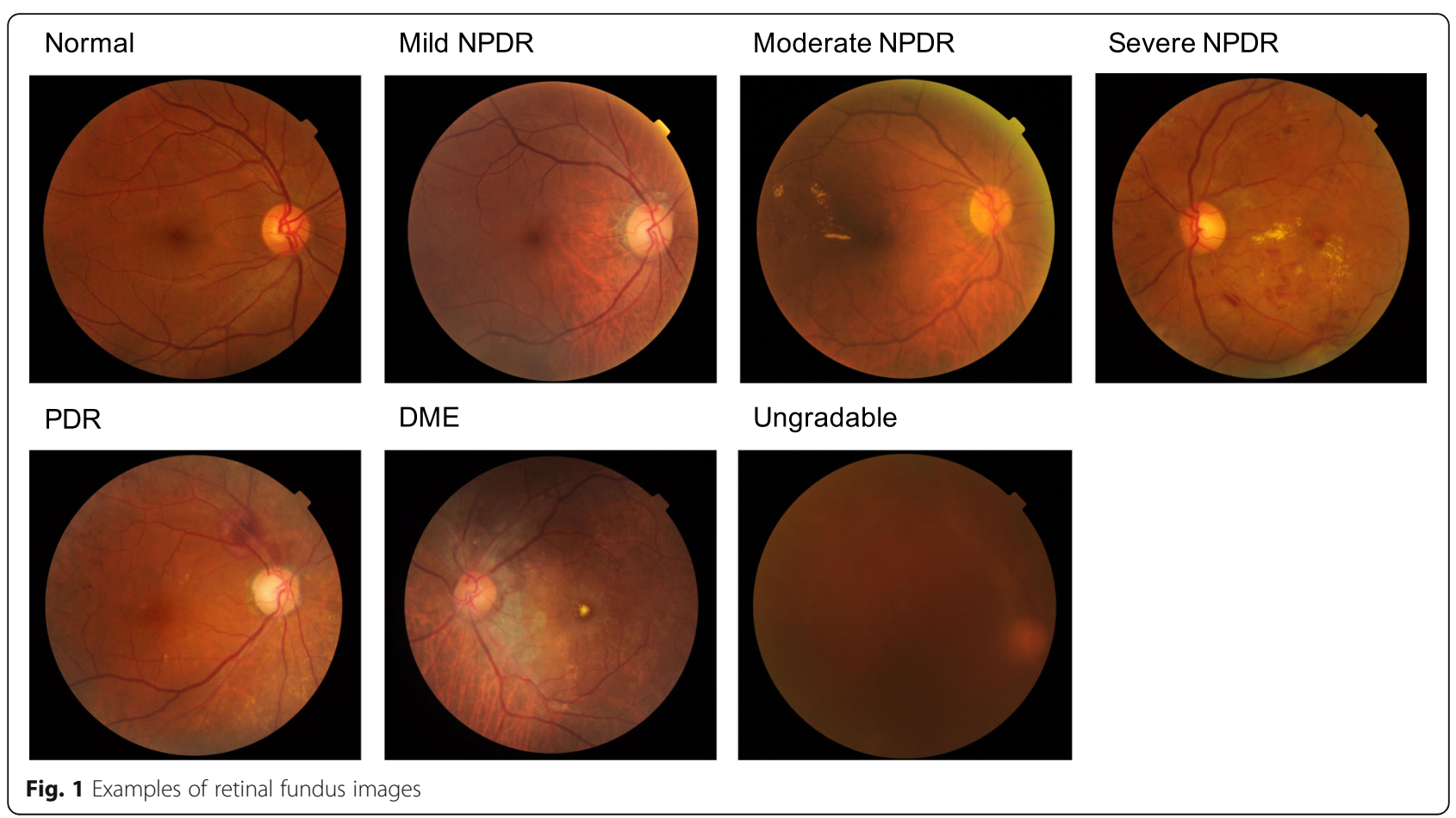

provides a comparison of retinal photographs obtained from different types of devices and capturing views. Table 1 summarises the major publications in retinal analysis using DL, separately describing standard multiple-field colour fundus photography, and the newer sub-modalities of ultra-wide field photography and smartphone-based photography. The approaches used for the various studies are also included in the table.

\section{Standard view}

Standard colour fundus photography provides a 30 to 50-degree image which includes the macula and optic

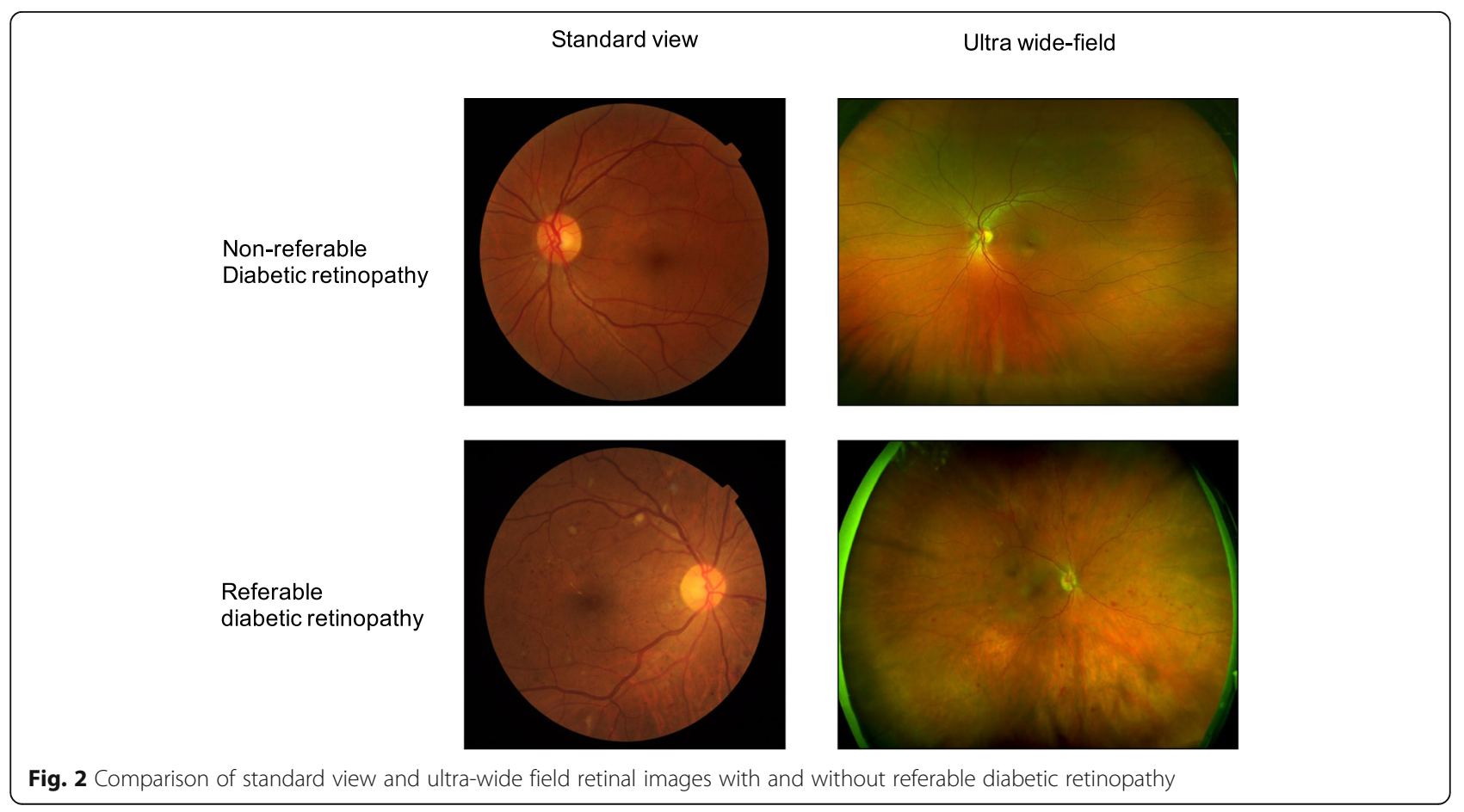


Table 1 Summary of the major publications in retinal analysis using DL, grouped by standard multiple-field fundus photography, ultra-wide field photography and smartphone-based photography

\begin{tabular}{|c|c|c|c|c|}
\hline Authors and year of publication & Approach & Training dataset & Validation datasets & Performance \\
\hline \multicolumn{5}{|l|}{ Standard view photography } \\
\hline \multirow[t]{8}{*}{ Gulshan et al. 2016 [35] } & \multirow[t]{8}{*}{ Inception-V3 network } & \multirow{8}{*}{$\begin{array}{l}\text { Public } \\
\text { EyePACS and Messidor-2 } \\
\text { (> 120,000 images) }\end{array}$} & \multirow{8}{*}{$\begin{array}{l}\text { Public } \\
\text { EyePACS-1 and Messidor-2 } \\
\text { (>10,000 images) }\end{array}$} & EyePACS-1 \\
\hline & & & & AUC: 0.99 \\
\hline & & & & Sensitivity: $90 \%$ \\
\hline & & & & Specificity: 98\% \\
\hline & & & & Messidor-2 \\
\hline & & & & AUC: 0.99 \\
\hline & & & & Sensitivity: $87 \%$ \\
\hline & & & & Specificity: 99\% \\
\hline \multirow[t]{3}{*}{ Abràmoff et al. 2016 [46] } & \multirow[t]{3}{*}{ AlexNet/NGG network } & \multirow{3}{*}{$\begin{array}{l}\text { Public } \\
\text { Messidor-2 }\end{array}$} & \multirow{3}{*}{$\begin{array}{l}\text { Public } \\
\text { Messidor-2 ( } 2000 \text { images) }\end{array}$} & AUC: 0.98 \\
\hline & & & & Sensitivity: $97 \%$ \\
\hline & & & & Specificity: $87 \%$ \\
\hline \multirow[t]{8}{*}{ Ting et al. 2017 [26] } & \multirow[t]{8}{*}{ VGGNet-19 network } & \multirow{8}{*}{$\begin{array}{l}\text { Proprietary } \\
\text { SiDRP 2010-2013 } \\
\text { (> 76,000 images) }\end{array}$} & \multirow{8}{*}{$\begin{array}{l}\text { Proprietary } \\
\text { SiDRP } 14-15 \text { and } 10 \text { others } \\
\text { (>112,000 images) }\end{array}$} & SiDRP 2014-2015 \\
\hline & & & & AUC: 0.93 \\
\hline & & & & Sensitivity: $91 \%$ \\
\hline & & & & Specificity: 92\% \\
\hline & & & & Others \\
\hline & & & & $\begin{array}{l}\text { AUC range: } 0.89 \\
\text { to } 0.98\end{array}$ \\
\hline & & & & $\begin{array}{l}\text { Sensitivity range: } \\
92 \text { to } 100 \%\end{array}$ \\
\hline & & & & $\begin{array}{l}\text { Specificity: } 76 \\
\text { to } 92 \%\end{array}$ \\
\hline \multirow[t]{8}{*}{ Gargeya et al. 2017 [47] } & \multirow[t]{8}{*}{ Customised CNN network } & \multirow{8}{*}{$\begin{array}{l}\text { Public EyePACS-1 } \\
\text { (> 75,000 images) }\end{array}$} & \multirow{8}{*}{$\begin{array}{l}\text { Public } \\
\text { EyePACS-1, Messidor-2,E- } \\
\text { Ophtha (> 17,000 images) }\end{array}$} & EyePACS-1 \\
\hline & & & & AUC: 0.97 \\
\hline & & & & Sensitivity: $94 \%$ \\
\hline & & & & Specificity: 96\% \\
\hline & & & & $\begin{array}{l}\text { Messidor-2 and } \\
\text { E-Ophtha }\end{array}$ \\
\hline & & & & $\begin{array}{l}\text { AUC range: } 0.83 \\
\text { to } 0.95\end{array}$ \\
\hline & & & & $\begin{array}{l}\text { Sensitivity range: } \\
74 \text { to } 93 \%\end{array}$ \\
\hline & & & & $\begin{array}{l}\text { Specificity range: } \\
87 \text { to } 94 \%\end{array}$ \\
\hline \multirow[t]{2}{*}{ Abràmoff et al. 2018 [34] } & \multirow[t]{2}{*}{ AlexNet/VGGNet network } & \multirow{2}{*}{$\begin{array}{l}\text { Public } \\
\text { Messidor-2 }\end{array}$} & \multirow{2}{*}{$\begin{array}{l}\text { Proprietary } \\
\text { Primary care sites } \\
\text { ( 900 patients) }\end{array}$} & Sensitivity: $87 \%$ \\
\hline & & & & Specificity: $91 \%$ \\
\hline \multirow[t]{2}{*}{ Keel et al. 2018 [48] } & \multirow[t]{2}{*}{ Inception-V3 network } & \multirow{2}{*}{$\begin{array}{l}\text { Public } \\
\text { LabelMe }(\sim 59,000)\end{array}$} & \multirow{2}{*}{$\begin{array}{l}\text { Proprietary } \\
\text { Endocrinology outpatient } \\
\text { services ( } 96 \text { patients) }\end{array}$} & Sensitivity: $92 \%$ \\
\hline & & & & Specificity: 94\% \\
\hline Kanagasingam et al. 2018 [49] & Inception-V3 network & $\begin{array}{l}\text { Public and proprietary } \\
\text { DiaRetDB1, EyePACS, } \\
\text { Australian tele-eye care } \\
\text { (30,000 images) }\end{array}$ & $\begin{array}{l}\text { Proprietary } \\
\text { Primary care ( } 200 \text { patients) }\end{array}$ & Sensitivity: 92\% \\
\hline \multirow[t]{2}{*}{ Gulshan et al. 2019 [50] } & \multirow[t]{2}{*}{ Inception-v4 network } & \multirow{2}{*}{$\begin{array}{l}\text { Public } \\
\text { EyePACS and Messidor-2 } \\
\text { (> 144,000 images) }\end{array}$} & \multirow{2}{*}{$\begin{array}{l}\text { Proprietary } \\
\text { Two eye hospitals } \\
(\sim 6000 \text { images })\end{array}$} & $\begin{array}{l}\text { AUC range: } 0.97 \\
\text { to } 0.98\end{array}$ \\
\hline & & & & $\begin{array}{l}\text { Sensitivity range: } \\
89 \text { to } 92 \%\end{array}$ \\
\hline
\end{tabular}


Table 1 Summary of the major publications in retinal analysis using DL, grouped by standard multiple-field fundus photography, ultra-wide field photography and smartphone-based photography (Continued)

\begin{tabular}{|c|c|c|c|c|}
\hline Authors and year of publication & Approach & Training dataset & Validation datasets & Performance \\
\hline & & & & $\begin{array}{l}\text { Specificity range: } \\
92 \text { to } 95 \%\end{array}$ \\
\hline \multirow[t]{3}{*}{ Raumviboonsuk et al. 2019 [51] } & \multirow[t]{3}{*}{ Inception-v4 network } & \multirow{3}{*}{$\begin{array}{l}\text { Public } \\
\text { EyePACS and Messidor-2 } \\
\text { (>120,000 images) }\end{array}$} & \multirow{3}{*}{$\begin{array}{l}\text { Proprietary } \\
\text { Hospitals and health centers } \\
(\sim 30,000 \text { images })\end{array}$} & AUC: 0.99 \\
\hline & & & & Sensitivity: $96.9 \%$ \\
\hline & & & & Specificity: $95.3 \%$ \\
\hline \multirow[t]{3}{*}{ Bellemo et al. 2019 [52] } & \multirow[t]{3}{*}{ VGGNet/ResNet network } & \multirow{3}{*}{$\begin{array}{l}\text { Proprietary } \\
\text { SiDRP 2010-2013 } \\
\text { (> 76,000 images) }\end{array}$} & \multirow{3}{*}{$\begin{array}{l}\text { Proprietary } \\
\text { Mobile screening unit } \\
\text { (> } 4000 \text { images) }\end{array}$} & AUC: 0.97 \\
\hline & & & & Sensitivity: $92 \%$ \\
\hline & & & & Specificity: 89\% \\
\hline \multicolumn{5}{|l|}{ Ultra-wide field photography } \\
\hline \multirow[t]{3}{*}{ Wang et al. 2018 [53] } & \multirow[t]{3}{*}{ EyeArt software } & \multirow[t]{3}{*}{-} & \multirow{3}{*}{$\begin{array}{l}\text { Proprietary } \\
\text { Eye clinics ( } 1500 \text { images) }\end{array}$} & AUC: 0.85 \\
\hline & & & & Sensitivity: $90 \%$ \\
\hline & & & & Specificity: 54\% \\
\hline \multirow[t]{3}{*}{ Nagasawa at al. 2019 [54] } & \multirow[t]{3}{*}{ VGGNet-16 network } & \multirow{3}{*}{$\begin{array}{l}\text { Proprietary Hospitals } \\
\text { (<400 images) }\end{array}$} & \multirow{3}{*}{$\begin{array}{l}\text { Proprietary } \\
\text { Hospitals (<400 images) }\end{array}$} & AUC: 0.97 \\
\hline & & & & Sensitivity: 95\% \\
\hline & & & & Specificity: 97\% \\
\hline \multicolumn{5}{|l|}{ Smartphone-based photography } \\
\hline \multirow[t]{2}{*}{ Rajalakshmi et al. 2018 [55] } & \multirow[t]{2}{*}{ EyeArt software } & \multirow[t]{2}{*}{-} & \multirow{2}{*}{$\begin{array}{l}\text { Proprietary } \\
\text { Tertiary care diabetes hospital } \\
\text { ( } \sim 300 \text { images })\end{array}$} & Sensitivity: 96\% \\
\hline & & & & Specificity: $80 \%$ \\
\hline \multirow[t]{2}{*}{ Natarajan et al. 2019 [56] } & \multirow[t]{2}{*}{$\begin{array}{l}\text { Remidio software } \\
\text { Inception-V3 network }\end{array}$} & \multirow{2}{*}{$\begin{array}{l}\text { Public and proprietary } \\
\text { EyePACS and hospitals } \\
\text { (>52,000 images) }\end{array}$} & \multirow{2}{*}{$\begin{array}{l}\text { Proprietary } \\
\text { Population-based screening } \\
\text { (> } 4000 \text { images) }\end{array}$} & $\begin{array}{l}\text { Sensitivity range: } \\
96 \text { to } 100 \%\end{array}$ \\
\hline & & & & $\begin{array}{l}\text { Specificity range: } \\
79 \text { to } 88 \%\end{array}$ \\
\hline \multirow[t]{3}{*}{ Rogers et al. 2019 [57] } & \multirow[t]{3}{*}{ Pegasus software } & \multirow[t]{3}{*}{-} & \multirow{3}{*}{$\begin{array}{l}\text { Public and proprietary } \\
\text { IDRiD and research laboratory } \\
\text { study (> } 6000 \text { images) }\end{array}$} & $\begin{array}{l}\text { AUC range: } 89 \\
\text { to } 99 \%\end{array}$ \\
\hline & & & & $\begin{array}{l}\text { Sensitivity range: } \\
82 \text { to } 93 \%\end{array}$ \\
\hline & & & & $\begin{array}{l}\text { Specificity range: } \\
82 \text { to } 94 \%\end{array}$ \\
\hline
\end{tabular}

nerve. It is widely used in clinical and trial settings as it provides relatively good documentation of DR. Multiple images can be manually overlapped to create a montage for example, 7 standard 30 degree colour fundus images may be combined to produce a 75 degree horizontal field of view [58]. With the addition of mydriasis, the proportion of ungradable photographs may be reduced from 26 to $5 \%(p<0.001)$ [59].

AI systems have generally been shown to be able to accurately detect DR from colour fundus photographs. During the early development and validation of the screening performance of DL systems, most scientific groups evaluated their CNN performances in developed countries, mostly on the United States population [35, 46, 47]. In 2016, Abràmoff et al. developed and enhanced a DL system which achieved a AUC of 0.98 and an achievable sensitivity and specificity of 96.8 and $87.0 \%$ in detecting referable DR (defined as moderate non-proliferative DR or worse, including diabetic macular oedema) on a publicly available colour fundus dataset (Messidor-2) [46]. Gulshan et al. also reported promising diagnostic performances of their DL system with an AUC of 0.99, and an achievable sensitivity and specificity of above 96 and 93\%, respectively, on two publicly available colour fundus datasets (EyePACS-1 and Messidor-2) [35]. Several other notable studies were conducted in the same year, as awareness of the promising abilities of DL in DR screening aroused the interest of the vision science and medical research communities [60-62].

In 2017, Gargeya and Leng customized a CNN model that achieved an AUC of 0.97 with 94\% sensitivity and 98\% specificity, on five-fold cross-validation using the EyePACS dataset [47]. They further tested it on two external datasets, achieving AUC scores of 0.94 and 0.95, respectively. Ting et al. then evaluated the performance of their DL system in detecting DR, using colour fundus images collected from a Singaporean national DR screening program, and achieved an AUC of 0.94 with 
an achievable sensitivity and specificity of 91 and $92 \%$ [26]. They further validated the system on 10 additional multi-ethnic multi-cohort multi-settings datasets with diabetes and achieved AUCs ranging from 0.89 to 0.98 . Concurrently, interest in DL continued to grow, with many noteworthy studies published [53, 63-68].

In 2018, IDX-DR software utilizing Alex/VGGNet features was validated with an external dataset [69] that was also approved for use by the US FDA, [34] having reported a sensitivity of $91 \%$ and specificity of $87 \%$ in a real-world clinical setting. Other pilot studies have also shown the applicability of such technologies in realworld settings and primary care $[48,49,70]$.

There has thus been much sustained interest regarding the application of DL systems for DR. [71-76] The most notable research direction in 2019 was arguably towards assessing the transferability of AI to other less-explored settings, particularly in developing countries. The Google AI group extended their works to Thailand and India. Ruamviboonsuk et al. reported promising sensitivity and specificity of 97 and 96\%, respectively, (AUC of 0.99) in a national screening program from local hospitals and health in Thailand [51]. In India, their DL system achieved a sensitivity and specificity of 89 and $92 \%$, respectively, (AUC of 0.96) on data from the Aravind Eye Hospital, and 92 and 95\%, respectively, (AUC of 0.98) on data from Sankara Nethralaya [50]. Bellemo et al. reported a promising sensitivity and specificity (92 and $89 \%$, respectively, with AUC of 0.97) for diagnosis in Zambia, a low middleincome African country [52]. In all the above developing countries, the DL systems' performance was either superior or comparable to that of human graders. This might provide an impetus for other countries of similar income levels to adopt DL systems for their routine national DR screening programmes [75].

Another notable trend has been the use of a DL system as an assistive tool for human graders. Sayres et al. investigated the use of heat maps generated by a DL system as a guidance system for human graders, which led to a significant improvement in diagnostic accuracy as compared to unassisted humans [77]. Keel et al. investigated a method to visualize the areas where their DL system focused in diagnosing DR. [78] Other applications concern the prediction of cardiovascular risk factors from colour fundus images, as well as the estimation of DR prevalence $[79,80]$. In addition, a promising field that might be explored is the use of DL for the generation of synthetic retinal images to overcome legal concerns and low disease prevalence [81].

\section{Ultra-wide field}

Ultra-wide field imaging allows examination of not only the central retinal area but also the peripheral zones, for up to a 200-degree view of the retina [82]; more than $80 \%$ of the total retinal surface can be captured in a single image. With its wide coverage, ultra-wide field imaging is able to detect predominantly peripheral lesions in eyes with DR, with more than $50 \%$ of the graded lesions present outside the seven standard Early Treatment Diabetic Retinopathy Study fields [83, 84]. The presence and increasing extent of predominantly peripheral lesions have been associated with an increased risk of DR progression. Therefore, the automated analysis of ultra-wide field images could be of value in DR screening, given the prognostic importance of peripheral lesions in predicting the progression to advanced disease [84].

In 2017, Levenkova et al. developed an algorithm for the automatic recognition of DR features, including bright (cotton wool spots and exudates) and dark lesions (microaneurysms and blot, dot and flame haemorrhages) in ultrawide field images [85]. The algorithm extracted DR features from grayscale and colour-composite UWF images, including intensity, histogram-of-gradient and local binary patterns. The best AUCs for bright and dark lesions are 94 and $95 \%$, respectively, achieved by a Support Vector Machine classifier. Wang et al. also evaluated performance of an automated AI algorithm for detecting referable DR, with $92 \% / 90 \%$ sensitivity with $50 \% / 54 \%$ specificity achieved for detecting referral-warranted retinopathy at the patient and eye levels, respectively [53]. More recently in 2019, Nagasawa et al. used ultra-wide field fundus images to detect treatment-naïve proliferative DR. Utilizing 378 photographic images to train the DL model, a high AUC of 0.97 with promising sensitivity of $94.7 \%$ and specificity of $97.2 \%$ was achieved [54].

\section{Smartphone-based}

Even though fundus cameras are commonly used in developed regions for DR screening, due to the high cost of equipment and lack of adequate number of trained ophthalmic technicians, deployment in rural areas with medically underserved patient populations remains limited [86]. In recent years, several solutions incorporating additional lens elements to smartphone cameras have been developed to provide affordable solutions and scalable approaches to widespread care.

In 2013, Prasanna et al. developed a smartphone-based decision support system attached to a handheld ophthalmoscope, for screening DR using sophisticated image analysis and ML techniques. It achieved an average sensitivity of $86 \%$ [87]. After a preliminary study [88], Rajalakshmi et al. assessed the role of an AI system for detection of DR and sight-threatening DR by colour fundus photography taken using smartphone-based retinal imaging system in 2018, and validated it against grading by ophthalmologists [55]. The AI system achieved $96 \%$ sensitivity and $80 \%$ specificity in detecting any DR, and 99\% sensitivity and $80 \%$ specificity in detecting sight- 
threatening DR with a kappa agreement of 0.78 and 0.75 , respectively. In 2019 , Wei et al. presented a realtime implementation of CNNs as a smartphone app to provide a low-cost alternative to fundus cameras equipped with lenses [89]. Natarajan et al. also evaluated the performance of another offline, smartphone-based AI system, for the detection of referable DR by using the images taken by the same smartphone-based retinal imaging system on different patient groups [56]. The sensitivity and specificity in diagnosing referable DR were 100 and $88 \%$, respectively, and in diagnosing any DR were 85 and $92 \%$, respectively, compared with ophthalmologist grading. Finally, Rogers et al. evaluated the performance of an AI system from images captured by a handheld portable fundus camera collected during a real-world clinical practice. Validation on the detection of proliferative DR resulted in an AUC of 0.92, with an AUC of 0.90 for referable DR. [57]

\section{Machine Learning Techniques \& Concepts}

State-of-the-art DL systems for DR classification generally may be understood in terms of the ML techniques and concepts involved. In particular, contributions by different groups may be analysed according to the choices made pertaining to each technique/concept. Here, we provide a broad overview of common techniques/concepts, and the trade-offs and considerations involved.

\section{Model architecture}

The DL model architecture is a major design choice, as the evidence on natural images strongly suggests that the model architecture used affects the classification performance level that may be attained, on the same training and validation data [35]. There has been constant innovation in terms of general-purpose endto-end deep network architectures in recent years [90], with some notable examples being LeNet, AlexNet, VGGNet, Inception, ResNet, DenseNet and SENet, roughly in chronological order of publication (Table 2).

However, for the medical imaging domain in particular, the declared performance of these architectures on largescale natural image classification may not always be the most relevant, due to other considerations. For one, the relatively small quantity of medical image data available may lead to overtraining and/or difficulties with training to convergence, with more-sophisticated and highercapacity models. As such, other than the careful application of transfer learning (covered later), older and simpler architectures may sometimes be favoured for particular applications. For example, the VGGNet architecture remains exceptionally suited for the extraction of intermediate features [91], while requiring relatively more weight parameters than other popular architectures [90].

Moreover, end-to-end classification is not the only paradigm for DL in DR screening. For instance, a hybrid approach would be to deploy DL models as low-level detectors that directly target various classes of lesions. Lim et al. trained models similar to LeNet on spatiallytransformed representations of candidate lesions proposed by a maximally-stable extremal region detector, [10] while Abràmoff et al.'s IDx-DR X2.1 used models inspired by AlexNet and VGGNet [46]. In these cases, the projected number and location of true lesions can either be directly matched against clinical reference standards, or the detector output vectors may be used as the input to a fusion algorithm that perfoms the final image-level classification.

Another notable consideration for model architectures would be the amount of computing resources required,

Table 2 Major deep learning model architecture families and characteristics. Note that there may be multiple variants (usually with different number of layers/parameters) within each architecture family

\begin{tabular}{|c|c|c|c|c|c|}
\hline Architecture family & Original year & Parameters & Layers & Module organization & Example application(s) \\
\hline AlexNet & 2012 & $\sim 60$ million & 8 & Convolutional, Max Pooling & $\begin{array}{l}\text { Abràmoff et al. [34], } \\
\text { Quellec et al. [66] }\end{array}$ \\
\hline VGGNet & 2014 & 180 million & 19 & Convolutional, Max Pooling & $\begin{array}{l}\text { Abràmoff et al. [34], } \\
\text { Quellec et al. [66], } \\
\text { Ting et al. [26], } \\
\text { Gargeya et al. [47], } \\
\text { Bellemo et al. [52] }\end{array}$ \\
\hline GoogLeNet (also Inception v1) & 2015 & $\sim 7$ million & 22 & Inception, Pool+Concat & Takahashi et al. [63] \\
\hline Inception (v3) & 2015 & $\sim 24$ million & 42 & Inception, Pool+Concat & $\begin{array}{l}\text { Gulshan et al. [35], } \\
\text { Krause et al. [30] }\end{array}$ \\
\hline ResNet & 2016 & $\sim 60$ million & 152 & Convolutional, Skip Connections & Bellemo et al. [52] \\
\hline Inception-ResNet (v2) & 2016 & 56 million & 164 & Residual Inception & - \\
\hline SqueezeNet & 2016 & 1.2 million(before pruning) & 14 & $\begin{array}{l}1 \times 1 \text { Convolutional, } \\
\text { Squeeze \& Expand Layers }\end{array}$ & - \\
\hline ResNeXt & 2017 & $\sim 25$ million & 50 & Convolutional (Grouped) & - \\
\hline DenseNet & 2017 & $\sim 20$ million & 201 & Dense, Transition & - \\
\hline
\end{tabular}


which is relevant for deployment on consumer devices such as smartphones, embedded systems, and on possibly less-powerful hardware in under-resourced regions. In general, the fewer the number of weight parameters involved in the model architecture, the quicker the inference, ceteris paribus. If the inference time is sufficiently quick, real-time analysis further becomes possible [92]. To this end, lightweight model architectures such as MobileNet [93] and ShuffleNet [94] have been designed for devices with limited computing power. Alternatively, model compression through pruning and parameter quantization may be done [95]. Given the medical implications of DR screening, however, any such trade-offs of performance for speed may need to be carefully considered.

\section{Ensembling}

Ensembling involves the combination of multiple independent ML classifier models, to produce a final classifier model that generally performs better than any of its constituent models. With DL models, ensembling is commonly and easily implemented by training multiple models - not necessarily of the same network architecture or inputs - separately, and then combining the outputs of these models during inference. Although regularization techniques such as dropout may be utilized during model training as an approximation to ensembling [96], models trained in this way nonetheless yield further performance gains when ensembled, in practice.
The number of models involved in the final ensemble is a trade-off between training/inference time and performance. Generally, the larger the number of independent models used, the better the performance, but with diminishing returns. For example, Gulshan et al. used an ensemble of ten Inception-v3 models [35], Ting et al. used an ensemble of two VGGNet-based models, although with differently pre-processed inputs [26], which was further extended with a ResNet model in Bellemo et al. [52]

Various methods have been employed for integrating the individual model outputs within an ensemble. Perhaps the most straightforward would be to take a linear average over these predictions, as was done for Gulshan et al. [35] and Ting et al. [26] More complex possibilities would include weighted ensembles [25] and the training of a further classifier model over the ensemble output values.

\section{Transfer learning}

Transfer learning is a method of adapting a model trained on some domain, to another domain (Fig. 3) [97]. For DL models in DR screening, the most prominent application of transfer learning has perhaps been in the finetuning of models that have already been pretrained on another classification task, such as ImageNet [98]. The reasoning behind such transfer learning is that the retinal image domain and the natural image domain share some similarities, especially for universal lowerlevel features such as corners and edges. Therefore, the

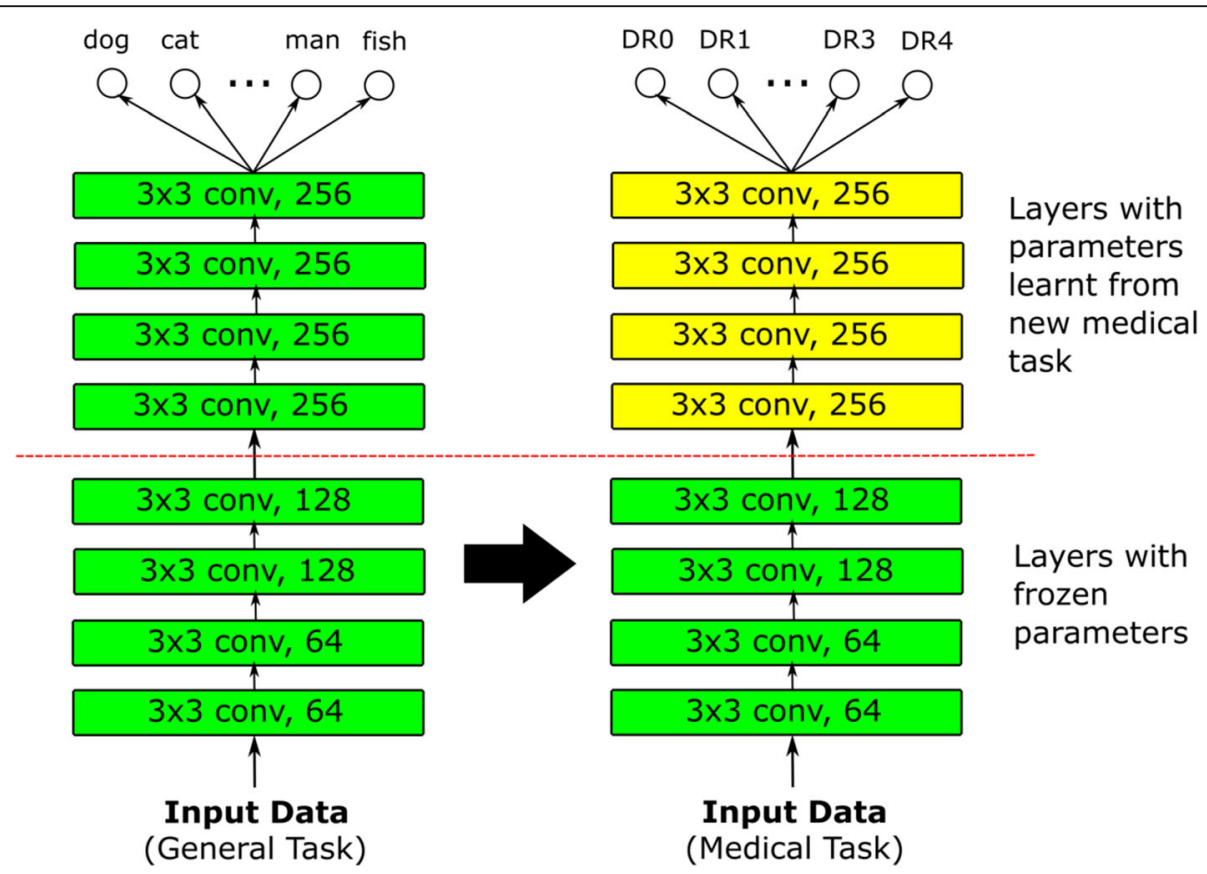

Fig. 3 Basic transfer learning method. A deep learning model is first trained on some general task. This trained model is then trained on the actual target medical task, possibly with the parameters for earlier layers representing low-level features frozen 
parameter weights from a natural image classification task should then serve as a good initialization for retinal image classification.

A major consideration for transfer learning with pretrained weights would be the policy by which these pretrained weights are finetuned with new retinal data. One possible choice would be to consider the pretrained weights merely as an initialization and proceed with training as per normal, allowing all weight values to be updated. At the other extreme, all pretrained weights are fixed, and the pretrained model is effectively employed as a feature extractor with only the output layer replaced, possibly by another classifier such as a random forest [47] or support vector machine [99]. Otherwise, the weights of any number of layers within the model architecture may be fixed, with the remainder updated; if so, it is generally the layers corresponding to lower-level features that are fixed. A previous survey on transfer learning in the medical domain by Tajbakhsh et al. suggests that although the use of pretrained weights made DL models more robust to the size of training sets, the optimal selection of layers to fix depends on the task at hand and has to be empirically determined [98].

\section{Weakly supervised and active learning}

A commonly encountered obstacle to training DL models for DR classification is a lack of annotated image data, particularly at the lesion level, since such detailed annotation was not typically required in clinical screening workflows. This made gathering sufficient lesion-level ground truth for hybrid DL implementations challenging. Although coarse-grained image-level grades were more widely available, it remained common to have large quantities of unlabelled retinal images for which no grades from human experts were available [100].

In such situations, weakly-supervised transductive learning becomes applicable. In transductive learning, an initial model trained on the labelled training data is used to classify the unlabelled training data. The originallyunlabelled training data now also becomes labelled, and may be used together with the originally-labelled training data to train an improved bootstrapped model [101].

Whether or not such transductive learning is employed, it is advisable to continually refine the trained model through active learning. Active learning presumes the presence of an oracle that can provide accurate answers to queries, which in the case of DR screening would be a human expert. However, there is an opportunity cost to consulting the oracle. As such, the goal of active learning is to intelligently select the most useful images for which to consult the oracle on, in the sense that the availability of accurate labels for these images would improve model performance to the greatest extent. One possible approach would be to select images for which the model is most uncertain [75].

\section{Label Modelling}

Another manifestation of weakly-supervised learning is the presence of imperfect or noisy labels. The presence of such imperfect labels is largely unavoidable in DR screening, with qualified human graders sometimes disagreeing with each other - or even themselves, from a previous session. Inter-grader kappa scores typically range from 0.40 to 0.65 in DR grading [102], and the implied disagreement may be resolved by majority decision, discussion between the graders, or external adjudication. Krause et al. conclude that rigorous adjudication of DR ground truth is important in developing DR models, since it allows for the principled correction of subtle errors from image artefacts and missed microaneurysms [30].

A further development by Guan et al. has been the modelling of individual graders with independent DL models, following the observation that the labelling of large DR datasets usually involves a large number of human graders, each of whom however grade only a relatively small subset of the dataset, with each image moreover also being graded by only a small subset of the human graders [102]. They found that modelling each human grader separately and averaging the predictions of these separate DL models in a weighted ensemble produced better performance than modelling the expected prediction of the average grader.

\section{Joint Learning}

DR may co-occur with other related eye diseases, and there is as such motivation to model its features together with those of other eye diseases. This joint or multitask learning involves training a DL model for multiple tasks simultaneously, and may induce beneficial regularization of intermediate representations, thus reducing overfitting [103]. González-Gonzalo et al. attempted the joint learning of referable DR and agerelated macular degeneration, and concluded that a jointly-trained DL model could perform comparably to human graders [104].

Joint learning may also be implemented for improving mid-level representations, in terms of optimizing for visual encodings and the final binary classifier at the same time, for multiple-instance learning [105]. This multipleinstance learning framework also allows for a degree of model interpretability by allowing the class of encoding instances to be explicitly considered during training. In this case, two neural networks are utilized to generate the mid-level representation encodings.

\section{Hyperparameter search \& optimization}

Other than the model weight parameters themselves, DL models involve a large number of hyperparameters, such as the initial learning rate, the learning rate decay schedule, the input batch size, etc. For DR screening 
applications, these hyperparameter settings are often borrowed directly from existing models, and whether these settings are the most appropriate for the DR screening domain may not be systematically explored. Sahlsten et al. is an example of work that investigates the image resolution parameter in detail [106].

The optimization of multiple hyperparameters is nontrivial, due to the number of hyperparameter combinations increasing exponentially with the number of individual hyperparameters. Although grid search over the hyperparameter space is commonly attempted, when the number of relevant hyperparameters is relatively small, random search [107] and sequential optimization algorithms [108] may also be attempted to more thoroughly examine possible model performance.

\section{Robustness}

Although DL models may be trained and validated on large datasets, it is difficult to be certain whether the datasets used can fully capture the potential variability of retinal images that may be encountered in future use. Differences may arise in the image acquisition process or population demographics that can render a trained DL model less effective on new data. Lim et al. demonstrated that the uncertainty of a DL model could be estimated by the standard deviation and entropy of the mean predictive distribution, on the stochastic batch normalization layers of a ResNet architecture, and that prediction error is correlated with high estimated uncertainty [75].

\section{Explainability}

A persistent obstacle against the uptake of AI systems in DR screening has been a lack of surface explainability [16]. In fact, the progression from handcrafted features and multi-stage classification to end-to-end deep learning has been accompanied by a concurrent loss of interpretability, in that humans could no longer examine the reasoning of the classifier, unlike previously where an image kernel could be inspected to determine why it had not matched with a microaneurysm, for instance.

This lack of interpretability has been mitigated somewhat through the development of various methods to extract saliency heatmaps from DL models, such as Grad-CAM [42] and integrated gradients [43]. These saliency heatmaps attempt to display the contribution of each image pixel or region to the final classification. This

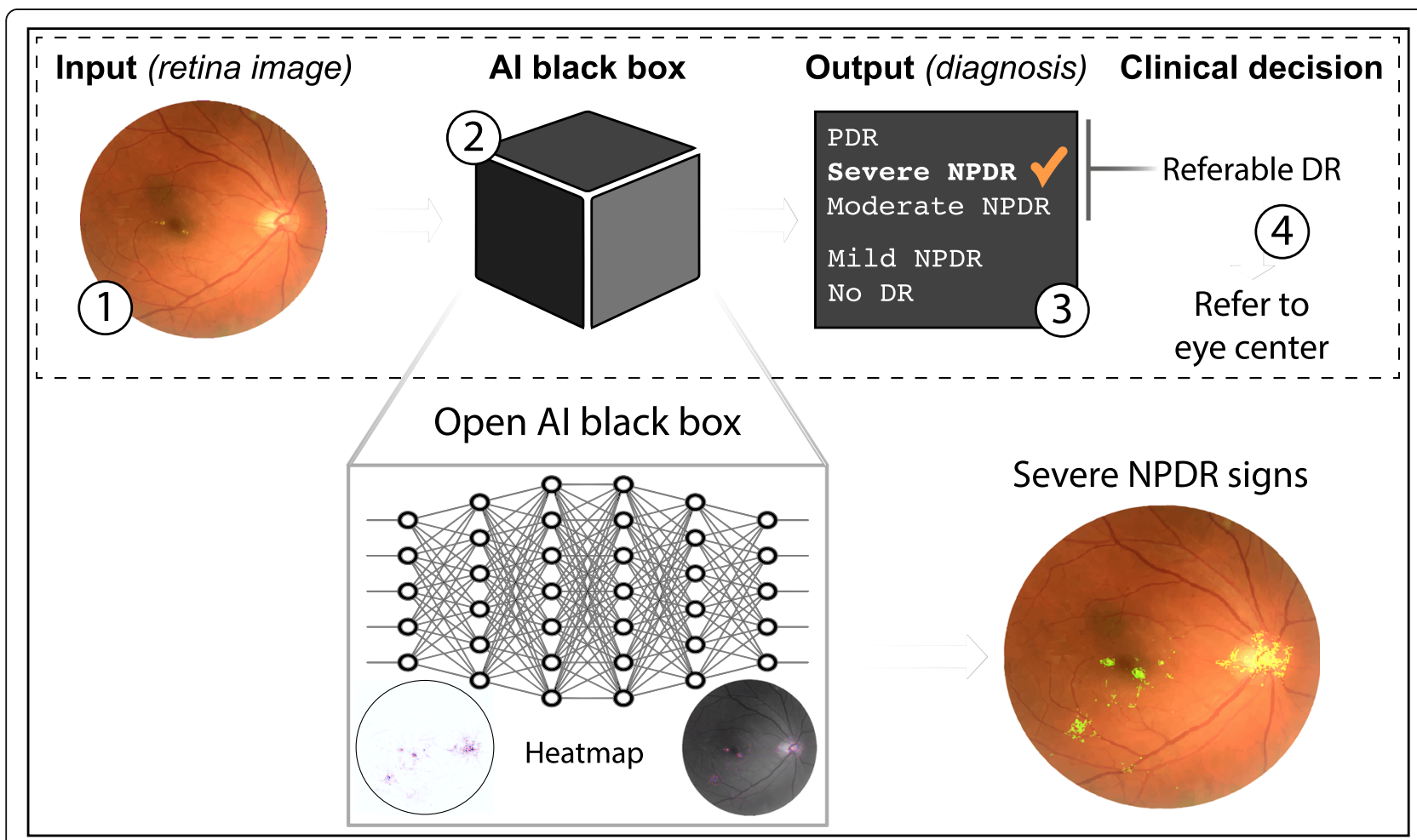

Fig. 4 Al flow for diabetic retinopathy. In the diabetic retinopathy screening domain, the Al implementation allows automated diagnosis and subsequent clinical decisions. In the example presented in the figure, the Al system would recommend referring the patient to the eye clinic because of the referable diagnosis for diabetic retinopathy. To allow researchers and clinicians determine how the Al model makes the decision, the heatmap attempts to display the contribution of each image pixel or region, to the final classification. Heatmaps open the 'black box' highlighting the areas in which the Al system is focusing on to build trust among practitioners and patients. Abbreviations: DR; diabetic retinopathy; NPDR: non-proliferative diabetic retinopathy; PDR: proliferative diabetic retinopathy 
allows researchers to retrospectively determine whether their DL models are making their decisions based on the expected image features, which in the DR screening domain would be various lesions such as microaneurysms, haemorrhages and hard exudates (Fig. 4).

A desire for greater interpretability has also seen renewed interest in hybrid methods that expose the intermediate goals of the classifier [109]. For example, Yang et al. implemented a two-stage DL model, which first classifies overlapping grid patches as containing lesions or not. The resulting weighted lesion map is then used as input to a second global DL model, to predict the image-level DR severity [110]. Wang et al. introduced a Zoom-in-Net architecture that purports to mimic the attentional behaviour of human graders, by allowing for suspicious regions to be focused on through additional learning on feature maps from the main network [111].

\section{Conclusions}

In this paper, we provided a broad overview of the major works and technical implementations involving DL techniques for DR diagnosis as an alternative tool for screening programmes. It emerged that, in the ophthalmology field, DL tools for DR show clinically acceptable diagnostic performance when using colour retinal fundus images. DL-based AI models are among the most promising solutions to tackle the burden of DR management in a comprehensive manner. However, future research is crucial to assess the potential clinical deployment, evaluate the costeffectiveness of different DL systems in the clinical practice and improve clinical acceptance.

\section{Abbreviations}

DR: Diabetic retinopathy; DL: Deep learning; Al: Artificial intelligence; CNN: Convolutional neural network; AUC: Area under the receiver operating characteristic curve

\section{Acknowledgements}

Not applicable.

\section{Authors' contributions}

$G L, V B$, and DT contributed to the initial drafting of the manuscript. GL, VB, $Y X, X Q, M Y$ and DT contributed to the critical review and final approval for this manuscript.

\section{Funding}

Funding from Research Grants Council-General Research Fund, Hong Kong (Ref: 14102418); National Medical Research Council Health Service Research Grant, Large Collaborative Grant, Ministry of Health, Singapore; the SingHealth Foundation; and the Tanoto Foundation.

Availability for data and materials

Not applicable.

Ethics approval and consent to participate

Not applicable.

\section{Consent for publication}

Not applicable.

\section{Competing interests}

Drs Daniel S.W. Ting and Gilbert Lim are co-inventors of a deep learning system for retinal diseases.

\section{Author details}

${ }^{1}$ School of Computing, National University of Singapore, Singapore, Singapore. ${ }^{2}$ Singapore Eye Research Institute, Singapore National Eye Centre, Singapore, Singapore. ${ }^{3}$ Duke-NUS Medical School, National University of Singapore, 11 Third Hospital Road Avenue, Singapore 168751, Singapore.

${ }^{4}$ Vitreo-Retinal Service, Singapore National Eye Center, 11 Third Hospital Road Avenue, Singapore 168751, Singapore. ${ }^{5}$ Artificial Intelligence in

Ophthalmology, Singapore Eye Research Institute, 11 Third Hospital Road Avenue, Singapore 168751, Singapore.

Received: 12 December 2019 Accepted: 10 March 2020

Published online: 14 April 2020

\section{References}

1. Moss SE, Klein R, Klein BE. The 14-year incidence of visual loss in a diabetic population. Ophthalmology. 1998;105(6):998-1003.

2. Yau JW, Rogers SL, Kawasaki R, Lamoureux EL, Kowalski JW, Bek T, et al. Global prevalence and major risk factors of diabetic retinopathy. Diabetes Care. 2012;35(3):556-64.

3. Cheung N, Mitchell P, Wong TY. Diabetic retinopathy. Lancet. 2010; 376(9735):124-36.

4. Ting DSW, Cheung GCM, Wong TY. Diabetic retinopathy: global prevalence, major risk factors, screening practices and public health challenges: a review. Clin Exp Ophthalmol. 2016:44(4):260-77.

5. Fogel AL, Kvedar JC. Artificial intelligence powers digital medicine. NPJ Digit Med. 2018:1:5.

6. Wong TY, Bressler NM. Artificial intelligence with deep learning technology looks into diabetic retinopathy screening. JAMA. 2016;316(22):2366-7.

7. Group ETDRSR. Early photocoagulation for diabetic retinopathy: ETDRS report number 9. Ophthalmology. 1991;98(Suppl 5):766-85.

8. Abràmoff MD, Niemeijer M, Suttorp-Schulten MS, Viergever MA, Russell SR, Van Ginneken B. Evaluation of a system for automatic detection of diabetic retinopathy from color fundus photographs in a large population of patients with diabetes. Diabetes Care. 2008;31(2):193-8.

9. Peto $T$, Tadros C. Screening for diabetic retinopathy and diabetic macular edema in the United Kingdom. Curr Diab Rep. 2012;12(4):338-45.

10. Lim G, Lee ML, Hsu W, Wong TY. Transformed representations for convolutional neural networks in diabetic retinopathy screening. In: AAAI Workshop: Modern Artificial Intelligence for Health Analytics. Quebec, 2014. pp. 21-5.

11. Lachure J, Deorankar A, Lachure S, Gupta S, Jadhav R. Diabetic retinopathy using morphological operations and machine learning. In: 2015 IEEE International Advance Computing Conference (IACC). Banglore, 2015. p. 617-22.

12. Prasad DK, Vibha L, Venugopal KR. Early detection of diabetic retinopathy from digital retinal fundus images. In: 2015 IEEE Recent Advances in Intelligent Computational Systems (RAICS). Trivandrum: IEEE; 2015. p. 240-5.

13. Scotland GS, McNamee P, Philip S, Fleming AD, Goatman KA, Prescott GJ, et al. Cost-effectiveness of implementing automated grading within the national screening programme for diabetic retinopathy in Scotland. $\mathrm{Br}$ J Ophthalmol. 2007:91(11):1518-23.

14. Scotland GS, McNamee P, Fleming AD, Goatman KA, Philip S, Prescott GJ, et al. Costs and consequences of automated algorithms versus manual grading for the detection of referable diabetic retinopathy. $\mathrm{Br} J$ Ophthalmol. 2010:94(6):712-9.

15. Trucco E, Ruggeri A, Karnowski T, Giancardo L, Chaum E, Hubschman JP, et al. Validating retinal fundus image analysis algorithms: issues and a proposal. Invest Ophthalmol Vis Sci. 2013;54(5):3546-59.

16. Ting DSW, Pasquale LR, Peng $L$, Campbell JP, Lee AY, Raman $R$, et al. Artificial intelligence and deep learning in ophthalmology. $\mathrm{Br} J$ Ophthalmol. 2019;103(2):167-75.

17. Cheung CY, Tang F, Ting DSW, Tan GSW, Wong TY. Artificial intelligence in diabetic eye disease screening. Asia Pac J Ophthalmol (Phila). 2019:8(2):158-64.

18. Sinthanayothin C, Boyce JF, Williamson TH, Cook HL, Mensah E, Lal S, et al. Automated detection of diabetic retinopathy on digital fundus images. Diabet Med. 2002;19(2):105-12. 
19. Usher D, Dumskyj M, Himaga M, Williamson TH, Nussey S, Boyce J. Automated detection of diabetic retinopathy in digital retinal images: a tool for diabetic retinopathy screening. Diabet Med. 2004;21(1):84-90.

20. Niemeijer M, Van Ginneken B, Staal J, Suttorp-Schulten MS, Abràmoff MD. Automatic detection of red lesions in digital color fundus photographs. IEEE Trans Med Imaging. 2005;24(5):584-92.

21. Staal J, Abràmoff MD, Niemeijer M, Viergever MA, Van Ginneken B. Ridgebased vessel segmentation in color images of the retina. IEEE Trans Med Imaging. 2004;23(4):501-9.

22. Lee A, Taylor P, Kalpathy-Cramer J, Tufail A. Machine learning has arrived! Ophthalmology. 2017;124(12):1726-8.

23. LeCun Y, Bengio Y, Hinton G. Deep learning. Nature. 2015;521(7553):436-44.

24. Bejnordi BE, Veta M, Van Diest PJ, Van Ginneken B, Karssemeijer N, Litjens G, et al. Diagnostic assessment of deep learning algorithms for detection of lymph node metastases in women with breast cancer. JAMA. 2017;318(22): 2199-210

25. Lakhani P, Sundaram B. Deep learning at chest radiography: automated classification of pulmonary tuberculosis by using convolutional neural networks. Radiology. 2017;284(2):574-82.

26. Ting DSW, Cheung CY, Lim G, Tan GSW, Quang ND, Gan A, et al. Development and validation of a deep learning system for diabetic retinopathy and related eye diseases using retinal images from multiethnic populations with diabetes. JAMA. 2017;318(22):2211-23.

27. Ting DSW, Yi PH, Hui F. Clinical applicability of deep learning system in detecting tuberculosis with chest radiography. Radiology. 2018;286(2):729-31.

28. Esteva A, Robicquet A, Ramsundar B, Kuleshov V, DePristo M, Chou K, et al. A guide to deep learning in healthcare. Nat Med. 2019;25(1):24-9.

29. Kapetanakis W, Rudnicka AR, Liew G, Owen CG, Lee A, Louw V, et al. A study of whether automated diabetic retinopathy image assessment could replace manual grading steps in the English National Screening Programme. J Med Screen. 2015;22(3):112-8.

30. Krause J, Gulshan V, Rahimy E, Karth P, Widner K, Corrado GS, et al. Grader variability and the importance of reference standards for evaluating machine learning models for diabetic retinopathy. Ophthalmology. 2018; 125(8):1264-72.

31. Nguyen HV, Tan GSW, Tapp RJ, Mital S, Ting DSW, Wong HT, et al. Costeffectiveness of a national telemedicine diabetic retinopathy screening program in Singapore. Ophthalmology. 2016;123(12):2571-80

32. Tufail A, Rudisill C, Egan C, Kapetanakis W, Salas-Vega S, Owen CG, et al. Automated diabetic retinopathy image assessment software: diagnostic accuracy and cost-effectiveness compared with human graders. Ophthalmology. 2017;124(3):343-51

33. Xie Y, Nguyen Q, Bellemo V, Yip MY, Lee XQ, Hamzah H, et al. CostEffectiveness Analysis of an Artificial Intelligence-Assisted Deep Learning System Implemented in the National Tele-Medicine Diabetic Retinopathy Screening in Singapore. Invest Ophthalmol Vis Sci. 2019;60(9):5471.

34. Abràmoff MD, Lavin PT, Birch $M$, Shah N, Folk JC. Pivotal trial of an autonomous Al-based diagnostic system for detection of diabetic retinopathy in primary care offices. NPJ Digit Med. 2018;1(1):39.

35. Gulshan V, Peng L, Coram M, Stumpe MC, Wu D, Narayanaswamy A, et al. Development and validation of a deep learning algorithm for detection of diabetic retinopathy in retinal fundus photographs. JAMA. 2016;316(22): 2402-10.

36. Grassmann F, Mengelkamp J, Brandl C, Harsch S, Zimmermann ME, Linkohr $B$, et al. A deep learning algorithm for prediction of age-related eye disease study severity scale for age-related macular degeneration from color fundus photography. Ophthalmology. 2018;125(9):1410-20.

37. Li Z, He Y, Keel S, Meng W, Chang RT, He M. Efficacy of a deep learning system for detecting glaucomatous optic neuropathy based on color fundus photographs. Ophthalmology. 2018;125(8):1199-206.

38. Brown JM, Campbell JP, Beers A, Chang K, Ostmo S, Chan RP, et al. Automated diagnosis of plus disease in retinopathy of prematurity using deep convolutional neural networks. JAMA Ophthalmol. 2018;136(7):803-10

39. Ting DSW, Peng L, Varadarajan AV, Keane PA, Burlina PM, Chiang MF, et al. Deep learning in ophthalmology: the technical and clinical considerations. Prog Retin Eye Res. 2019;72:100759.

40. Ribeiro MT, Singh S, Guestrin C. Why should I trust you?: Explaining the predictions of any classifier. In: Proceedings of the 2016 Conference of the North American Chapter of the Association for Computational Linguistics: Demonstrations. San Diego, 2016. p. 97-101.https://doi.org/ 10.18653/v1/N16-3020.
41. Lundberg SM, Lee SI. A unified approach to interpreting model predictions. In: Advances in Neural Information Processing Systems. Long Beach, 2017. p. 4765-74.

42. Selvaraju RR, Cogswell M, Das A, Vedantam R, Parikh D, Batra D. Grad-cam: visual explanations from deep networks via gradient-based localization. Int J Comput Vis. 2020;128:336-59. https://doi.org/10.1007/s11263-019-01228-7 .

43. Sundararajan M, Taly A, Yan Q. Axiomatic attribution for deep networks. arXiv:1703.01365, 2017

44. Goh JKH, Cheung CY, Sim SS, Tan PC, Tan GSW, Wong TY. Retinal imaging techniques for diabetic retinopathy screening. J Diabetes Sci Technol. 2016; 10(2):282-94.

45. Abràmoff MD, Garvin MK, Sonka M. Retinal imaging and image analysis. IEEE Rev Biomed Eng. 2010;3:169-208.

46. Abràmoff MD, Lou Y, Erginay A, Clarida W, Amelon R, Folk JC, et al. Improved automated detection of diabetic retinopathy on a publicly available dataset through integration of deep learning. Invest Ophthalmol Vis Sci. 2016;57(13):5200-6.

47. Gargeya R, Leng T. Automated identification of diabetic retinopathy using deep learning. Ophthalmology. 2017:124(7):962-9.

48. Keel S, Lee PY, Scheetz J, Li Z, Kotowicz MA, Maclsaac RJ, et al. Feasibility and patient acceptability of a novel artificial intelligence-based screening model for diabetic retinopathy at endocrinology outpatient services: a pilot study. Sci Rep. 2018:8(1):4330.

49. Kanagasingam Y, Xiao D, Vignarajan J, Preetham A, Tay-Kearney ML, Mehrotra A. Evaluation of artificial intelligence-based grading of diabetic retinopathy in primary care. JAMA Netw Open. 2018;1 (5):e182665.

50. Gulshan V, Rajan RP, Widner K, Wu D, Wubbels P, Rhodes T, et al. Performance of a deep-learning algorithm vs manual grading for detecting diabetic retinopathy in India. JAMA Ophthalmol. 2019. https:/doi.org/10.1001/jamaophthalmol.2019.2004.

51. Raumviboonsuk P, Krause J, Chotcomwongse P, Sayres R, Raman R, Widner K, et al. Deep learning versus human graders for classifying diabetic retinopathy severity in a nationwide screening program. NPJ Digit Med. 2019;2:25.

52. Bellemo V, Lim ZW, Lim G, Nguyen QD, Xie Y, Yip MY, et al. Artificial intelligence using deep learning to screen for referable and visionthreatening diabetic retinopathy in Africa: a clinical validation study. Lancet Digital Health. 2019;1(1):e35-44.

53. Wang K, Jayadev C, Nittala MG, Velaga SB, Ramachandra CA, Bhaskaranand $M$, et al. Automated detection of diabetic retinopathy lesions on ultrawidefield pseudocolour images. Acta Ophthalmol. 2018;96(2):e168-73.

54. Nagasawa T, Tabuchi H, Masumoto H, Enno H, Niki M, Ohara Z, et al. Accuracy of ultrawide-field fundus ophthalmoscopy-assisted deep learning for detecting treatment-naïve proliferative diabetic retinopathy. Int Ophthalmol. 2019;39(10):2153-9.

55. Rajalakshmi R, Subashini R, Anjana RM, Mohan V. Automated diabetic retinopathy detection in smartphone-based fundus photography using artificial intelligence. Eye (Lond). 2018;32(6):1138-44.

56. Natarajan S, Jain A, Krishnan R, Rogye A, Sivaprasad S. Diagnostic accuracy of community-based diabetic retinopathy screening with an offline artificial intelligence system on a smartphone. JAMA Ophthalmol. 2019. https://doi. org/10.1001/jamaophthalmol.2019.2923.

57. Rogers T, Gonzalez-Bueno J, Franco RG, Star EL, Marín DM, Vassallo J, et al. Evaluation of an Al system for the detection of diabetic retinopathy from images captured with a handheld portable fundus camera: the MAILOR Al study. arXiv preprint arXiv:190806399. 2019.

58. Baumal CR, Duker JS. Current management of diabetic retinopathy: Elsevier Health Sciences; 2017.

59. Murgatroyd H, Ellingford A, Cox A, Binnie M, Ellis J, MacEwen C, et al. Effect of mydriasis and different field strategies on digital image screening of diabetic eye disease. Br J Ophthalmol. 2004;88(7):920-4.

60. Pratt H, Coenen F, Broadbent DM, Harding SP, Zheng Y. Convolutional neural networks for diabetic retinopathy. Procedia Computer Science. 2016;90:200-5.

61. Colas E, Besse A, Orgogozo A, Schmauch B, Meric N, Besse E. Deep learning approach for diabetic retinopathy screening. Acta Ophthalmol. 2016;94. https://doi.org/10.1111/j.1755-3768.2016.0635

62. Doshi D, Shenoy A, Sidhpura D, Gharpure P. Diabetic retinopathy detection using deep convolutional neural networks. In: 2016 International Conference on Computing, Analytics and Security Trends (CAST). Pune: IEEE; 2016. p. 261-6.

63. Takahashi $H$, Tampo H, Arai Y, Inoue Y, Kawashima H. Applying artificial intelligence to disease staging: deep learning for improved staging of diabetic retinopathy. PLoS One. 2017;12(6):e0179790.

64. Gegundez-Arias ME, Marin D, Ponte B, Alvarez F, Garrido J, Ortega C, et al. A tool for automated diabetic retinopathy pre-screening based on retinal image computer analysis. Comput Biol Med. 2017;88:100-9. 
65. Xu K, Feng D, Mi H. Deep convolutional neural network-based early automated detection of diabetic retinopathy using fundus image. Molecules. 2017;22(12). https://doi.org/10.3390/molecules22122054

66. Quellec G, Charrière K, Boudi Y, Cochener B, Lamard M. Deep image mining for diabetic retinopathy screening. Med Image Anal. 2017;39:178-93.

67. Abbas Q, Fondon I, Sarmiento A, Jiménez S, Alemany P. Automatic recognition of severity level for diagnosis of diabetic retinopathy using deep visual features. Med Biol Eng Comput. 2017;55(11):1959-74.

68. Choi JY, Yoo TK, Seo JG, Kwak J, Um TT, Rim TH. Multi-categorical deep learning neural network to classify retinal images: a pilot study employing small database. PLoS One. 2017;12(11):e0187336.

69. Van Der Heijden AA, Abramoff MD, Verbraak F, van Hecke MV, Liem A, Nijpels $G$. Validation of automated screening for referable diabetic retinopathy with the IDx-DR device in the Hoorn Diabetes Care System. Acta Ophthalmol. 2018:96(1):63-8.

70. Verbraak FD, Abramoff MD, Bausch GC, Klaver C, Nijpels G, Schlingemann RO, et al. Diagnostic accuracy of a device for the automated detection of diabetic retinopathy in a primary care setting. Diabetes Care. 2019;42(4):651-6.

71. de La Torre J, Valls A, Puig D. A deep learning interpretable classifier for diabetic retinopathy disease grading. Neurocomputing. 2019. In press. https://doi.org/10.1016/j.neucom.2018.07.102.

72. Li T, Gao Y, Wang K, Guo S, Liu H, Kang H. Diagnostic assessment of deep learning algorithms for diabetic retinopathy screening. Inf Sci. 2019;501:511-22.

73. Liu YP, Li Z, Xu C, Li J, Liang R. Referable diabetic retinopathy identification from eye fundus images with weighted path for convolutional neural network. Artif Intell Med. 2019;99:101694.

74. Raman R, Srinivasan S, Virmani S, Sivaprasad S, Rao C, Rajalakshmi R. Fundus photograph-based deep learning algorithms in detecting diabetic retinopathy. Eye (Lond). 2019:33(1):97-109.

75. Lim ZW, Lee ML, Hsu W, Wong TY. Building Trust in Deep Learning System towards automated disease detection. In: Proceedings of the AAAl Conference on Artificial Intelligence. 2019. p. 9516-21. https://doi.org/10. 1609/aaai.v33i01.33019516.

76. Yip MY, Lim G, Bellemo V, Xie Y, Lee XQ, Nguyen Q, et al. Effect of image compression and number of fields on a deep learning system for detection of diabetic retinopathy. Invest Ophthalmol Vis Sci. 2019;60(9):1438.

77. Sayres R, Taly A, Rahimy E, Blumer K, Coz D, Hammel N, et al. Using a deep learning algorithm and integrated gradients explanation to assist grading for diabetic retinopathy. Ophthalmology. 2019;126(4):552-64.

78. Keel S, Wu J, Lee PY, Scheetz J, He M. Visualizing deep learning models for the detection of referable diabetic retinopathy and glaucoma. JAMA Ophthalmol. 2019;137(3):288-92.

79. Poplin R, Varadarajan AV, Blumer K, Liu Y, McConnell MV, Corrado GS, et al. Prediction of cardiovascular risk factors from retinal fundus photographs via deep learning. Nat Biomed Eng. 2018;2(3):158-64.

80. Ting DS, Cheung CY, Nguyen Q, Sabanayagam C, Lim G, Lim ZW, et al. Deep learning in estimating prevalence and systemic risk factors for diabetic retinopathy: a multi-ethnic study. NPJ Digit Med. 2019;2:24.

81. Bellemo V, Burlina P, Yong L, Wong TY, Ting DSW. Generative adversarial networks (GANs) for retinal fundus image synthesis. In: Asian Conference on Computer Vision. 2018. p. 28-302.

82. Aiello LP, Odia I, Glassman AR, Melia M, Jampol LM, Bressler NM, et al. Comparison of early treatment diabetic retinopathy study standard 7-field imaging with ultrawide-field imaging for determining severity of diabetic retinopathy. JAMA Ophthalmol. 2019;137(1):65-73.

83. Ghasemi Falavarjani K, Wang K, Khadamy J, Sadda SR. Ultra-wide-field imaging in diabetic retinopathy; an overview. J Curr Ophthalmol. 2016;28(2):57-60.

84. Silva PS, Cavallerano JD, Haddad NMN, Kwak H, Dyer KH, Omar AF, et al. Peripheral lesions identified on ultrawide field imaging predict increased risk of diabetic retinopathy progression over 4 years. Ophthalmology. 2015; 122(5):949-56.

85. Levenkova A, Sowmya A, Kalloniatis M, Ly A, Ho A. Automatic detection of diabetic retinopathy features in ultra-wide field retinal images. In: Medical Imaging 2017: Computer-Aided Diagnosis; International Society for Optics and Photonics. 2017: 101341M. https://doi.org/10.1117/12.2253980.

86. Fenner BJ, Wong RLM, Lam WC, Tan GSW, Cheung GCW. Advances in retinal imaging and applications in diabetic retinopathy screening: a review. Ophthalmol Ther. 2018;7(2):333-46.

87. Prasanna P, Jain S, Bhagat N, Madabhushi A. Decision support system for detection of diabetic retinopathy using smartphones. In: 2013 7th
International Conference on Pervasive Computing Technologies for Healthcare and Workshops. Venic: IEEE; 2013. p. 176-9.

88. Rajalakshmi R, Arulmalar S, Usha M, Prathiba V, Kareemuddin KS, Anjana RM, et al. Validation of smartphone based retinal photography for diabetic retinopathy screening. PLoS One. 2015;10(9):e0138285.

89. Wei H, Sehgal A, Kehtarnavaz N. A deep learning-based smartphone app for real-time detection of retinal abnormalities in fundus images. In: Real-Time Image Processing and Deep Learning 2019. Int Soc Opt Photonics. 2019; 10996:1099602

90. Canziani A, Paszke A, Culurciello E. An analysis of deep neural network models for practical applications. arXiv preprint arXiv:160507678. 2016.

91. Jing $Y$, Yang $Y$, Feng Z, Ye J, Yu Y, Song M. Neural style transfer: a review. IEEE Trans Vis Comput Graph. 2019. https://doi.org/10.1109/TVCG.2019.2921336.

92. Chen P, Gadepalli K, MacDonald R, Liu Y, Kadowaki S, Nagpal K, et al. An augmented reality microscope with real-time artificial intelligence integration for cancer diagnosis. Nat Med. 2019;25(9):1453-7.

93. Howard AG, Zhu M, Chen B, Kalenichenko D, Wang W, Weyand T, et al. Mobilenets: Efficient convolutional neural networks for mobile vision applications. arXiv preprint arXiv:170404861. 2017.

94. Zhang X, Zhou X, Lin M, Sun J. Shufflenet: an extremely efficient convolutional neural network for mobile devices. In: Proceedings of the IEEE Conference on Computer Vision and Pattern Recognition. Salt Lake City, 2018. p. 6848-56.

95. Cheng Y, Wang D, Zhou P, Zhang T. A survey of model compression and acceleration for deep neural networks. arXiv preprint arXiv:171009282. 2017.

96. Baldi P, Sadowski PJ. Understanding dropout. In: Advances in neural information processing systems. 2013.

97. Pan SJ, Yang Q. A survey on transfer learning. IEEE Trans Knowl Data Eng. 2009;22(10):1345-59.

98. Tajbakhsh N, Shin JY, Gurudu SR, Hurst RT, Kendall CB, Gotway MB, et al. Convolutional neural networks for medical image analysis: full training or fine tuning? IEEE Trans Med Imaging. 2016;35(5):1299-312.

99. Burlina PM, Joshi N, Pekala M, Pacheco KD, Freund DE, Bressler NM. Automated grading of age-related macular degeneration from color fundus images using deep convolutional neural networks. JAMA Ophthalmol. 2017;135(11):1170-6.

100. Lim G, Hsu W, Lee ML, Ting DSW, Wong TY. Technical and clinical challenges of A.I. in retinal image analysis. In: Trucco E, MacGillivray T, Xu Y, editors. Computational retinal image analysis:tools, applications, and perspectives, Elsevier-MICCAI Society Book Series. Academic Press; 2019. p. 445-66.

101. Arnold A, Nallapati R, Cohen WW. A comparative study of methods for transductive transfer learning. In: Seventh IEEE International Conference on Data Mining Workshops (ICDM). Omaha, 2007. p. 77-82.

102. Guan MY, Gulshan V, Dai AM, Hinton GE. Who said what: Modeling individual labelers improves classification. In: Thirty-Second AAAI Conference on Artificial Intelligence. 2018. arXiv:1703.08774v2.

103. Caruana R. Multitask learning. Mach Learn. 1997;28(1):41-75.

104. González-Gonzalo C, Sánchez-Gutiérrez V, Hernández-Martínez P, Contreras I, Lechanteur YT, Domanian A, et al. Evaluation of a deep learning system for the joint automated detection of diabetic retinopathy and age-related macular degeneration. arXiv preprint arXiv:190309555. 2019.

105. Costa P, Galdran A, Smailagic A, Campilho A. A weakly-supervised framework for interpretable diabetic retinopathy detection on retinal images. IEEE Access. 2018;6:18747-58.

106. Sahlsten J, Jaskari J, Kivinen J, Turunen L, Jaanio E, Hietala K, et al. Deep Learning Fundus Image Analysis for Diabetic Retinopathy and Macular Edema Grading. arXiv preprint arXiv:190408764. 2019.

107. Bergstra J, Bengio Y. Random search for hyper-parameter optimization. J Mach Learn Res. 2012;13(1):281-305.

108. Bergstra JS, Bardenet R, Bengio Y, Kégl B. Algorithms for hyper-parameter optimization. In: Advances in neural information processing systems. 2011. p. 2546-54.

109. Lim G, Hsu W, Lee ML. Intermediate goals in deep learning for retinal image analysis. In: Asian Conference on Computer Vision. Cham: Springer; 2018. p. 276-81.

110. Yang Y, Li T, Li W, Wu H, Fan W, Zhang W. Lesion detection and grading of diabetic retinopathy via two-stages deep convolutional neural networks. In: International Conference on Medical Image Computing and ComputerAssisted Intervention. Cham: Springer; 2017. p. 533-40.

111. Wang Z, Yin Y, Shi J, Fang W, Li H, Wang X. Zoom-in-net: deep mining lesions for diabetic retinopathy detection. In: International Conference on Medical Image Computing and Computer-Assisted Intervention. Cham: Springer; 2017. p. 267-75. 